\title{
Tissue-resident natural killer cells exacerbate tubulointerstitial fibrosis by activating transglutaminase 2 and syndecan- 4 in a model of aristolochic acid-induced nephropathy
}

\author{
Yu Mee Wee ${ }^{1}$, Heounjeong Go ${ }^{2}$, Monica Young Choi ${ }^{1}$, Hey Rim Jung ${ }^{1}$, Yong Mee Cho ${ }^{2}$, Young Hoon Kim ${ }^{3}$, Duck Jong Han ${ }^{3}$ \\ E Sung Shin ${ }^{1,3, *}$ \\ ${ }^{1}$ Department of Asan Institute for Life Science, Asan Medical Center, Seoul 05535, ${ }^{2}$ Department of Pathology, Asan Medical Center, \\ University of Ulsan College of Medicine, Seoul $05535,{ }^{3}$ Division of Kidney and Pancreas Transplantation, Department of Surgery, Asan \\ Medical Center, University of Ulsan College of Medicine, Seoul 05535, Korea
}

\begin{abstract}
Despite reports suggesting that tissue-resident natural killer (trNK) cells cause ischemic kidney injury, their contribution to the development of tubulointerstitial fibrosis has not been determined. This study hypothesized that the depletion of trNK cells may ameliorate renal fibrosis by affecting transglutaminase 2/syndecan-4 interactions. Aristolochic acid nephropathy (AAN) was induced in $\mathrm{C} 57 \mathrm{BL} / 6$ mice as an experimental model of kidney fibrosis. The mice were treated with anti-asialo GM1 (ASGM1) or anti-NK1.1 antibodies to deplete NK cells. Although both ASGM1 and NK1.1 antibodies suppressed renal NKp46 ${ }^{+}$DX5 ${ }^{+}$NK cells, renal NKp46 ${ }^{+}$DX5 ${ }^{-}$ cells were resistant to suppression by ASGM1 or NK1.1 antibodies during the development of tubulointerstitial fibrosis in the AAN-induced mouse model. Western blot analysis showed that both antibodies increased the expression of fibronectin, transglutaminase 2, and syndecan-4. These findings indicate that trNK cells played an exacerbating role in tubulointerstitial fibrosis by activating transglutaminase 2 and syndecan-4 in the AAN-induced mouse model. [BMB Reports 2019; 52(9): 554-559]
\end{abstract}

\section{INTRODUCTION}

The renal fibrosis is one of the final common pathway of a chronic kidney diseases (CKDs) and nephropathy. Renal fibrosis is initated by an excessive accumulation of extracellular components, inducing to distroy the kidney

${ }^{*}$ Corresponding author. Tel: +82-2-3010-3964; Fax: +82-2-30104182; E-mail: sshin@amc.seoul.kr

https://doi.org/10.5483/BMBRep.2019.52.9.193

Received 23 August 2018, Revised 29 October 2018, Accepted 26 December 2018

Keywords: Aristolochic acid nephropathy, Syndecan-4, Tissue-resident natural killer cell, Transglutaminase 2, Tubulointerstitial fibrosis function (1).

Aristolochic acid injection (AAI) was first reported to cause CKD in a report from Belgium. Huang et al. observed that AAI induced the severe reduction of peritubular capillaries, resulting in hypoxia and tubular cell death, leading to tubular atrophy. Consequently, AAl induced irreversible tubule damage causing interstitial fibrosis and tubular atrophy (IFTA) (2).

Transglutaminase 2 (TG2) catalyzes the cross-linking of protein in a calcium-dependent manner and (3), plays a central role in renal fibrosis. Increased extracellular TG2 enhances crosslink formation, contributing to the accumulation of extracellular matrix and the development of renal fibrosis (4). In addition, the heparan sulfate proteoglycan, syndecan-4 (Sdc4), was shown to be involved in extracellular trafficking and the cell surface targeting of profibrogenic TG2 in vivo and in vitro, suggesting that targeting the TG2-Sdc4 interaction may provide a specific interventional strategy for the treatment of chronic kidney disease (CKD) $(5,6)$.

Natural killer (NK) cells play a critical role in the early stages of innate immune responses against cells infected by pathogens, such as virus and microbe, and agaist tumor cells (7). Based on differences in trafficking and tissue retention, NK cells were recently shown to consist of two distinct subsets, tissue-resident NK cells (trNK) and conventional circulating NK (cNK) cells, NKp $46^{+} \mathrm{DX} 5^{-}$and $\mathrm{NKp} 46^{+} \mathrm{DX} 5^{+}$respectively (8). In addition to differences in trafficking, trNK and cNK cells showed distinct charateristics in cytokine production and cell surface proteins involved in cellular adhesion function and recognition of target cells $(9,10)$. Under homeostatic conditions, mouse kidneys were found to contain a significant fraction of trNK cells compared with cNK cells and anti-ASGM1 treatment resulted in a robust and selective depletion of cNK cells, while the trNK cells were largely left intact (10). In addition to these findings, trNK cells have been reported to be INF- $\gamma$-producing CD56 ${ }^{\text {bright }}$ NK cells, associated with interstitial fibrosis and poor clinical outcomes (11). Furthermore, INF- $\gamma$ produced by NK cell induces TG2

ISSN: 1976-670X (electronic edition)

Copyright (c) 2019 by the The Korean Society for Biochemistry and Molecular Biology

(c) This is an open-access article distributed under the terms of the Creative Commons Attribution Non-Commercial License (http://creativecommons.org/licenses/by-nc/4.0) which permits unrestricted non-commercial use, distribution, and reproduction in any medium, provided the original work is properly cited. 
production and activation (12). These findings support the idea that trNK cells facilitate the development and progression of renal fibrosis following IFN- $\gamma$-induced TG2 production and/or TG2-Sdc4 interaction.

This study assessed whether trNK cells were stimulators of profibrotic factors, such as TG2, Sdc4, and TGF- $\beta$. Accordingly, the role of NK cells in tubulointerstitial fibrosis was analyzed using an aristolochic acid nephropathy (AAN) model in mice.

\section{RESULTS}

Effects of NK cell-depleting antibodies on the proportions of splenic cNK and trNKcells in AAN-induced mice

NK cell depletion and renal fibrosis (AAN) were induced in mice by treatment with anti-ASGM1 or anti-NK1.1 antibodies and AAI injections, respectively (Fig. 1A). To assess the effects of both NK cell-depleting antibodies on each subset of NK cells in the spleen, the absolute number of leukocytes was evaluated by flow cytometry before the development of AAN. The lymphocytes were divided into $\mathrm{CD}^{+}{ }^{+} \mathrm{T}$ cells, $\mathrm{CD} 19^{+} \mathrm{B}$ cells,

A
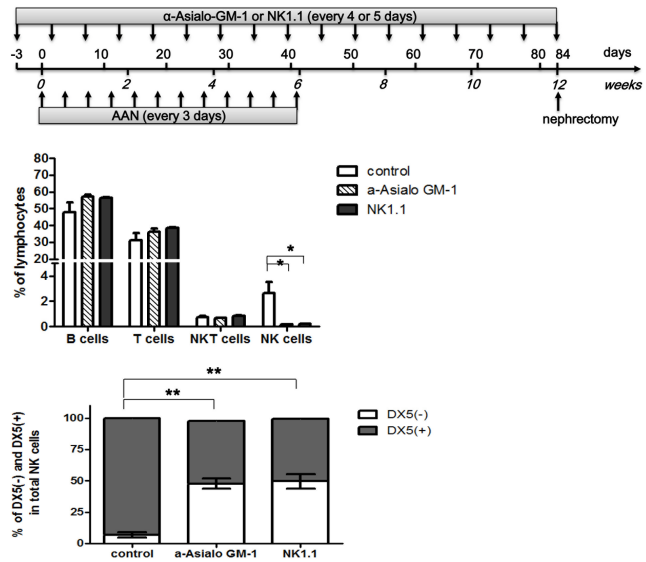

Fig. 1. Time schedule of $\mathrm{NK}$ cell depletion and $\mathrm{AAl}$-induced kidney fibrosis. (A) To induce AAN, AAI was injected into mice on day 0 and once every three days thereafter for six weeks, followed by housing under standard laboratory conditions for six weeks to establish chronic renal fibrosis. NK cells were depleted by injecting ASGM-1 or NK1.1 antibodies three days prior to AAI injection and once every four or five days thereafter for 12 weeks. Splenectomies was performed at six weeks and nephrectomies at 12 weeks. (B) Flow cytometric analysis of splenic lymphocyte types after injection of NK cell-depleting antibodies. T cells were defined as $\mathrm{CD}^{+}{ }^{+} \mathrm{CD} 19^{-} \mathrm{NKp} 46^{-}$ events, whereas B cells were defined as $\mathrm{CD} 3^{-} \mathrm{CD} 19^{+} \mathrm{NKp} 46^{-}$events. NK cells were defined as CD3 ${ }^{-} \mathrm{CD}_{19}{ }^{-} \mathrm{Nkp} 46^{+}$events and NKT cells as $\mathrm{CD}^{+} \mathrm{CD}^{-} 9^{-} \mathrm{NKp} 46^{+}$events. (C) DX5 subtype analysis of splenic NK cells in mice injected with or without NK cell-depleting antibodies. Antibody treatment enriched the population of $\mathrm{CD} 3^{+} \mathrm{CD} 19^{-} \mathrm{NKp} 46^{+} \mathrm{DX} 5^{-}$ $\mathrm{NK}$ cells compared to $\mathrm{CD} 3^{+} \mathrm{CD} 19^{-} \mathrm{NKp} 46^{+} \mathrm{DX} 5^{+} \mathrm{NK}$ cells. The results represent the mean $+\mathrm{SD}$ for five animals. ${ }^{*} \mathrm{P}<0.05$. AAN, aristolochic acid nephropathy; AAI, aristolochic acid I; ASGM-1, anti-asialo GM-1. ${ }^{*} \mathrm{P}<0.05, * * \mathrm{P}<0.005$.
$\mathrm{CD}^{+}{ }^{+} \mathrm{CD} 19^{-} \mathrm{NKp} 6^{+} \mathrm{NKT}$ cells, and $\mathrm{CD} 3^{-} \mathrm{CD}^{-} 9^{-} \mathrm{NKp} 46^{+} \mathrm{NK}$ cells. NK cells were significantly depleted by treatment with anti-ASGM1 or anti-NK1.1 antibodies, however, these antibodies did not affect the number of T, B, or NKT cells (Fig. 1B). Following treatment with anti-ASGM1 or anti-NK1.1 antibodies, the proportion of $\mathrm{NKp} 46^{+} \mathrm{DX} 5^{-}$(trNK) cells was higher, while the proportion of $\mathrm{NKp} 46^{+} \mathrm{DX} 5^{+}$(cNK) cells was significantly lower than in control mice spleens (Fig. 1C).

\section{Effects of NK cell-depleting antibodies on renal cNK and trNK cells in AAN-induced mice}

To assess the effects of both NK cell-depleting antibodies on each subset of NK cells in the spleen and kidney, the absolute number of leukocytes was evaluated by flow cytometry six and 12 weeks after the development of AAN in mice. cNK was the major splenic NK cell subtype and it was markedly decreased by NK cell-depleting antibodies, regardless of AAI treatment or the timing of the antibody injections. Whereas a population of trNK cells was not depleted by treatment with either anti-ASGM1 or anti-NK1.1 antibodies (Fig. 2A and 2B). Unlike the splenic NK cells, similar numbers of CNK and trNK cells were present in the control kidneys. Most of the cNK cells were depleted by NK cell-depleting antibodies, as in the spleen. The percentage of trNK cells in the kidneys decreased after AAI treatment or NK-depleting antibody injection, but the decrease was not statistically significant. However, trNK cells were resistant to antibody-mediated depletion when they were co-treated with AAl at six and 12 weeks (Fig. 2C and 2D). These findings indicate that NK depletion regimens using either anti-NK1.1 or anti-ASGM1 did not deplete trNK cells
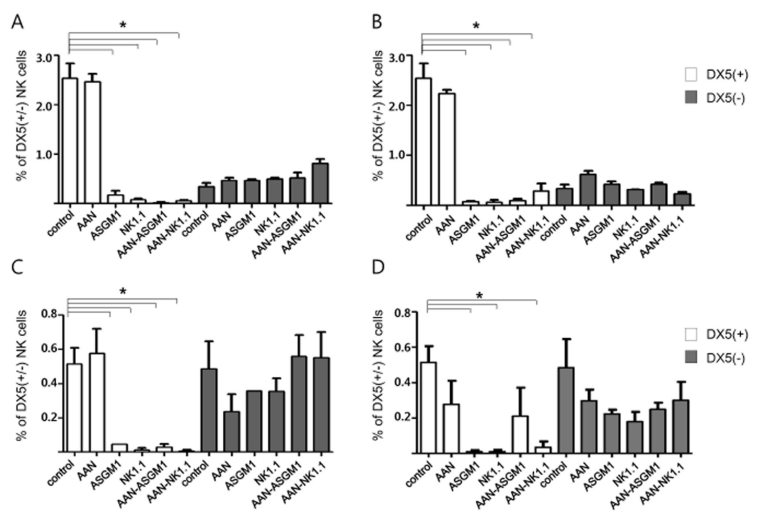

Fig. 2. Flow cytometric analysis of cNK and trNK cells in mice spleen and kidney tissues. After AAN induction and NK depletion, the frequencies of trNK cells (DX5 $5^{-}$NK: $\square$ ) to cNK cells (DX5 ${ }^{+} \mathrm{NK}$ : $\square$ ) were analyzed in each group at six (A) and 12 (B) weeks in splenic tissue and $6(\mathrm{C})$ and 12 (D) weeks in kidney tissue. Unlike the splenic NK cells, a similar number of cNK and trNK cells were present in the kidney cells. Compared to cNK cells, trNK cells were resistant to antibody-mediated depletion in both tissue types. The results represent the mean of 10 to 12 animals in each group. $* P<0.005$. 
during tubulointerstitial fibrosis progression.

\section{Effects of anti-NK1.1 or anti-ASGM1 antibodies on tubulointerstitial fibrosis in AAN-induced mice}

Weight gain did not differ significantly in the six groups of mice. However, kidney weights were significantly lower in the AAN-only group than in the control group $(0.179 \pm 0.009$ vs $0.218 \pm 0.011$ gm, $P<0.001)$. In contrast, kidney weights did not differ significantly in the control group compared to mice treated with anti-ASGM1 or anti-NK1.1 antibodies. AAI treatment tended to reduce kidney weights, whereas NK-depleting antibodies did not (data not shown).

Because the AAN model is a chronic nephropathy model, tubulointerstitial fibrosis had not developed six weeks after the AAI injections. Upon histological examination of the 12-week kidneys, H\&E, MT, and PAS stains showed the progressive development of tubulointerstitial fibrosis in the AAN mouse model, despite a fibrosis grade of 1 . Most of the AAI-induced fibrotic areas were located in the outer cortex because AAl-induced kidney disease is marked in the medulla and outer cortex (2). The kidneys of the AAN group showed enlarged interstitia, lumina, and glomeruli. Perivascular inflammation was also detected in this group, even though the inflammation grade indicated an early stage of the disease. At this time, collagen deposition and tubulointerstitial fibrosis did not differ significantly between the control group and either the ASGM1 or the NK1.1 group. However, the AAN-ASGM1 and AAN-Nk1.1 groups demonstrated remarkable tubuloin-

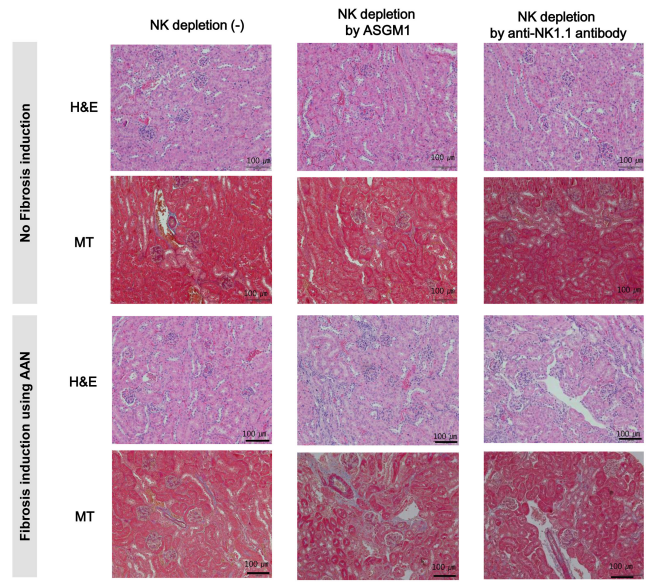

Fig. 3. Histomorphological photomicrographs of renal histology in each group. H\&E staining showed inflammatory cells in the tubulointerstitial space in the AAN-ASGM1 and AAN-NK1.1 groups. MT staining showed that most AAl-induced fibrotic areas were located in the outer cortex. All kidney specimens were graded for tubulointerstitial fibrosis as mild $(10 \%-25 \%)$, moderate $(25 \%-50 \%)$, or severe $(50 \%-100 \%)$ tubular atrophy and interstitial fibrosis was graded as 1, 2, or 3. Tubulointerstitial fibrosis was seen in the AAN, AAN-ASGM1, and AAN-NK1.1 groups. H\&E, hematoxylin \& eosin; MT, Masson's Trichrome; original magnification x 200. terstitial fibrosis characteristics. Compared with the control group, they had irregular kidney surfaces and thin cortices. Also, perivascular inflammation was increased and visible between the tubule and the cortical junction (Fig. 3). These findings suggest that tubulointerstitial fibrosis was exacerbated by NK cell-depleting antibodies during AAN development.

Increased expression of TG2 and Sdc4 in AAN-induced mice The expression levels of TG2 and Sdc4 were examined by Western blotting. Compared to the AAN group, the TG2 intensity was significantly higher in the AAN-ASGM1 and AAN-NK1.1 groups 12 weeks after the initiation of AAN treatment (Fig. 4A). Furthermore, the expression of Sdc4 was significantly higher in the AAN-ASGM1 group than in the AAN group. Similarly, the intensity of Sdc4 in the AAN-NK1.1 group was higher than in the AAN group, although the difference did not reach statistical significance (Fig. 4B). Moreover, the expression of fibronectin, the ultimate product of fibrosis, was significantly higher in the AAN-ASGM1 group than in the AAN group (Fig. 4D). In contrast, the expression of IFN- $\gamma$, one of the most important mediators of NK cells, was significantly higher in the ASGM1 group compared to other groups. Unexpectedly, the expression of IFN- $\gamma$ was decreased in all AAl-treated groups (AAN, AAN-ASGM1, and AAN-NK1.1) compared to the antibody-treated groups. These findings suggest that IFN- $\gamma$ was not associated with the progression of AAN in normal kidneys. However, IFN- $\gamma$ produced by the trNK cells may have contributed to the fibrosis progression that
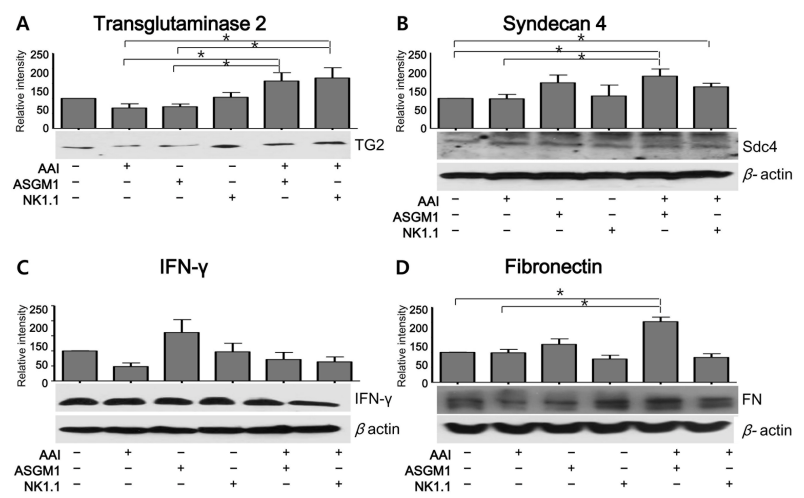

Fibronectin

Fig. 4. Western blot analysis of protein expression during fibrosis in each group. Representative and relative band intensities of Western blots for total TG2 (A), syndecan 4 (B), IFN- $\gamma(C)$, and fibronectin (D) in mice kidneys. Compared to normal mice, AAN mice treated with ASGM1 or NK1.1 antibodies showed significant increases in TG2, syndecan 4, and fibronectin, but not IFN- $\gamma$. Each protein was densitometrically analyzed and normalized to $\beta$-actin. Changes in the expression of each protein were assessed by dividing the level of expression in each experimental group by the level in the control group. The results represent the mean \pm SD for 10 animals. Western bands show representative findings from three different experiments. To detect the expression of TG2 (A) and IFN- $\gamma(\mathrm{C})$, the membrane was cut in two. Therefore, $(\mathrm{A})$ and $(\mathrm{C})$ had same amount of $\beta$-actin. ${ }^{*} \mathrm{P}<0.05$ 
occurred when the cNK cells were decreased by NK cell-depleting antibodies (Fig. 4C). We also examined the expression of TG2, Sny4, fibronectin, and IFN- $\gamma$ by Western blotting six weeks after starting AAN treatment. Significant differences were not detected at that time (data not shown).

\section{DISCUSSION}

The role of NK cells in different organ is reported that modulatory inflammatory responses is often complex and sometimes even paradoxical (13-16). The role of NK cells in kidney disease is also complex and remains unclear. In this study, NK cell depletion was performed to identify the role of trNK cells as important mediators of tubulointerstitial fibrosis. Depletion of NK cells, either by anti-ASGM1 or anti-NK1.1 antibody treatment is a method frequently used to evaluate the roles of NK cells in a wide range of immune-related pathophysiological processes. The NK1.1 receptor, a glycoprotein expressed on NK cells, and subsets of $\gamma \delta \mathrm{T}$, NKT, and CD8 T cells is expressed in a few mouse strains, such a C57BL/6. Asialo-GM1, a glycolipid highly expressed on NK cells, basophils, and subsets of $\gamma \delta \mathrm{T}, \mathrm{NKT}$, and CD8 T cells is expressed in multiple mouse strains, including B6, BALB/C, and DBA (17). Victorino et al. reported that the ASGM1 expression in trNK cells was reduced relative to that in $\mathrm{CNK}$ cells. Consequently, anti-ASGM-1 treatment resulted in the robust depletion of cNKs, leaving trNKs largely intact, whereas anti-NK1.1 effectively depleted both cNK and trNK cells (10). This study showed that these antibodies could deplete cNK cells during tubulointerstitial fibrosis induced by AAI. However, neither antibody completely depleted trNK cells from the kidneys of AAN-induced mice. Specifically, trNK cells still remained in the kidneys following treatment with AAI or either antibody. The results suggest that these antibodies may have contributed to the exacerbation of tubulointerstitial fibrosis in this AAN-induced mouse model.

Although few previous studies have assessed the effects of NK cell infiltration on renal fibrosis, NK cell infiltration into other organs, such as the liver and lungs, may protect against fibrosis progression, a protective effect that may be mediated by IFN- $\gamma$. For example, the production of endogenous IFN- $\gamma$ following lung injury has been shown to limit the extent of tissue remodeling and fibrosis and NK cells being an important source of IFN- $\gamma$. (18). Furthermore, NKp $46^{+} \mathrm{NK}$ cells were recently reported to attenuate metabolism-induced hepatic fibrosis by regulating macrophage activation in mice (4). We found that the trNK cells that infiltrated mice kidney tissue were resistant to depletion by both the anti-ASGM1 and anti-NK1.1 antibodies and were associated with the progression of tubulointerstitial fibrosis in the AAN mouse model. trNK cells have previously been shown to represent an immature form of NK cells and to be non-cytolytic, whereas mature NK cells are cytolytic (19). Our findings showed that a subset of NK cells resident in mice kidney tissue could exacerbate tubulointerstitial fibrosis. Unexpectedly, the relative intensity of IFN- $\gamma$ was decreased in all AAI treated groups but not in antibody treated groups (Fig. 4C). These findings suggest that IFN- $\gamma$ was not associated with the progression of $\mathrm{AAN}$ in normal mice kidneys. However, IFN- $\gamma$ produced by trNK cells may have contributed to fibrosis progression when the cNK cells were depleted by NK cell-depleting antibodies.

We previously reported that intragraft $\mathrm{CD}_{56}{ }^{+}$cells in kidney transplantation biopsy specimens were associated with interstitial fibrosis and poor clinical outcomes (20). In the present study, we demonstrated that trNK cells were resistant to NK cell depletion, not only by anti-ASGM1 but by anti-NK1.1 antibodies. Additionally, this subset of NK cells was associated with the aggravation of tubulointerstitial fibrosis. Notably, trNK cells in the AAN model were associated with increased intensities of TG2 and Sdc4 profibrotic factors. TG2 cell surface availability has been reported to depend on the trapping of TG2 by heparan sulfate chains of the transmembrane heparan sulfate proteoglycan, Sdc4 (6). Additionally, target cell surface heparan sulfate proteoglycans are recognized by NKp30 and NKp46, with further recognition and subsequent lysis by NK cells, which are dependent on the 6-O-sulfation and $\mathrm{N}$-acetylation states of the glucose building blocks (21). Based on our data, non-depleted trNK cells were associated with INF- $\gamma$ production, although this association was not statistically significant (Fig. 4C). Because INF- $\gamma$ produced by NK cell induces TG2 production (12), trNK cells may, therefore, exacerbate tubulointerstitial fibrosis by interactions with Sdc4 and subsequently, with TG2.

In summary, this study showed that a significant proportion of NK cells in the AAN-induced mouse model remained in the kidney tissue following treatment with anti-ASGM1 or anti-NK1.1 antibodies. This subset of NK cells was responsible for the progression of tubulointerstitial fibrosis. In addition, our results indicate a significant correlation between trNK cells and profibrotic factors, such as Sdc4 and TG2. Further experiments are required to determine the mechanism by which trNK cells regulate Sdc4 and TG2, and to identify strategies to inhibit the interaction between trNK cells and Sdc4/TG2, thereby preventing tubulointerstitial fibrosis.

\section{MATERIALS AND METHODS}

\section{Antibodies}

ASGM1 and NK1.1 antibodies were obtained from Wako Pure Chemicals (Osaka, Japan) and Bio X Cell (West Lebanon, $\mathrm{NH}$, USA), respectively. Purified rabbit anti-Sdc4, -IFN- $\gamma$, -TGF- $\beta 1$, and -TG2 antibodies were obtained from Abcam (Cambridge, MA, USA). Rat anti-CD49b (DX5, PE-conjugated) and rabbit anti-CD335 (NKp46, FITC-conjugated), anti-CD3 (PerCP-Cy5.5conjugated), and anti-CD19 (APC-conjugated) antibodies were obtained from BD Biosciences (San Jose, CA, USA). 


\section{Experimental animals}

Inbred C57BL/6] (B6) mice were purchased from the Jungang Laboratories (Seoul, Korea). All mice were maintained in a specific pathogen-free facility and housed under standard laboratory conditions of $22^{\circ} \mathrm{C} \pm 2^{\circ} \mathrm{C}$ and relative humidity of $55 \pm 5 \%$ with $12 \mathrm{~h}$ light/dark cycles. This study was reviewed and approved by the Institutional Animal Care and Use Committee (IACUC) of Asan Institute for Life Sciences, Asan Medical Center. The committee abides by the Institute of Laboratory Animal Resources (ILAR) guide. This study complies with the National Institutes of Health Guide for the Care and Use of Laboratory Animals.

\section{NK cell depletion and AAN induction}

Eight-week-old male C57BL/6 mice were used for NK cell depletion and aristolochic acid I (AAI, Sigma-Aldrich, St Louis, USA)-induced nephropathy. The mice were classified into six groups of 10-12 mice each: control, AAN only (AAN), NK cell depletion by ASGM1 only (ASGM1), NK cell depletion by anti-NK1.1 antibody only (NK1.1), AAN with NK cell depletion by ASGM1 (AAN-ASGM1), and AAN with NK cell depletion by anti-NK1.1 antibody (AAN-NK1.1). Progressive tubulointerstitial fibrosis was induced by the intraperitoneal injection of $3 \mathrm{mg} / \mathrm{kg} \mathrm{AAl}$ every three days for six weeks. NK cells were depleted by treating C57BL/6) mice with $100 \mu \mathrm{l}$ anti-NK1.1 antibody or $100 \mu \mathrm{g}$ anti-ASGM-1 antibody three days prior to AAI injection. The antibodies were injected every four or five days for 12 weeks. Anti-mouse IgG or anti-rabbit IgG was injected into the control group (both antibodies), NK1.1 group (mouse IgG), or ASGM1 group (anti-rabbit IgG). NK cell depletion was verified by measuring the percentage of $\mathrm{NK}$ cells $\left(\mathrm{CD} 45^{+} \mathrm{CD} 3^{-} \mathrm{NKp} 46^{+}\right)$five days after the last injection of the depleting antibody by flow cytometry (Fig. 1A). The mice in each group underwent splenectomies and nephrectomies at six weeks and 12 weeks.

\section{Histopathology of kidney tissue}

Twelve weeks after the induction of AAN, all kidney tissues were removed and fixed in $10 \%$ natural buffered formalin, processed, and embedded in paraffin. Sections $(4 \mu \mathrm{m})$ were stained with hematoxylin and eosin (H\&E), Masson trichrome (MT), and periodic acid-Schiff (PAS). All kidney specimens were graded for tubulointerstitial fibrosis by two renal pathologists. Tubular atrophy was graded as mild (10\%-25\%), moderate $(25 \%-50 \%)$, or severe $(50 \%-100 \%)$ and interstitial fibrosis was graded as 1, 2, or 3 .

\section{Flow cytometry analysis of splenic or renal lymphocytes}

The splenocytes were isolated by centrifugation on a Ficoll gradient and washed with Roswell Park Memorial Institute (RPMI) 1640 medium (Invitrogen, Carlsbad, CA, USA) media containing $5 \%(\mathrm{v} / \mathrm{v})$ fetal bovine serum (FBS, Invitrogen) (22). To isolate kidney leukocytes, the kidneys were dissociated using a Multi-Tissue Dissociation Kit 2 (Miltenyi Biotec,
Teterow, Germany) according to the manufacturer's instructions. Briefly, a mouse kidney was transferred into a $60 \mathrm{~mm}$ dish and dissected into several pieces. Tissue slices were transferred into a gentleMACS $C$ tube containing the enzyme mix. Using the gentleMACS $37^{\circ} \mathrm{C}$ Multi E_01 program, the tissues were dissociated into single cells. The dissociated tissues were transferred to conical tubes and centrifuged at $200 \mathrm{~g}$ for 10 $\min$. The cells were resuspended in RPMI and applied to a cell strainer $(100 \mu \mathrm{m})$ placed onto a conical tube.

For flow cytometry analysis, spleen or kidney cells were resuspended at concentrations of $3 \times 10^{5}$ cells/100 $\mu \mathrm{l}$, followed by the addition of $4 \mu \mathrm{l}$ of fluorescent-labeled antibodies, PerCp-Cy5.5-CD3, APC-CD19, FITC-NKp46, and PE-DX5. The cells were incubated on ice for $30 \mathrm{~min}$ and washed twice with phosphate buffered saline (PBS) containing $2 \%$ FBS. T cells were defined as $\mathrm{CD} 3^{+} \mathrm{CD} 19^{-} \mathrm{NKp} 46^{-}$events, whereas $\mathrm{B}$ cells were defined as $\mathrm{CD} 3^{-} \mathrm{CD} 19^{+} \mathrm{NKp} 46^{-}$events. $\mathrm{NK}$ cells were defined as $\mathrm{CD} 3^{-} \mathrm{CD} 19^{-} \mathrm{Nkp} 46^{+}$events and NKT cells as $\mathrm{CD} 3^{+} \mathrm{CD} 19^{-} \mathrm{NKp} 46^{+}$events. trNK and cNK cells were identified by DX5 ${ }^{-}$and DX5 ${ }^{+}$events, respectively.

\section{Western blot analysis}

Kidney tissue was minced on a mortar with $2 \mathrm{ml}$ liquid $\mathrm{N}_{2}$ and added to a radioimmunoprecipitation assay (RIPA) buffer containing a protease inhibitor cocktail (Sigma-Aldrich, St. Louis, MO, USA). The proteins were extracted by incubation, vortexing, and centrifugation $\left(15,000 \mathrm{~g}, 25 \mathrm{~min}\right.$ at $\left.4^{\circ} \mathrm{C}\right)$. The protein concentrations of the supernatants were measured using the bicinchoninic acid protein assay (BCA) (Pierce, Rockford, IL, USA). An equal volume of $2 \mathrm{X}$ sodium dodecyl sulfate (SDS) sample buffer (Sigma-Aldrich) was added to 20 $\mu \mathrm{g}$ protein and the proteins were resolved by $8 \%-10 \%$ SDS-polyacrylamide gel electrophoresis and transferred to polyvinylidene fluoride membranes. The membranes were blocked for $1 \mathrm{~h}$ with 5\% BSA, then incubated with anti-Sdc4, anti-IFN- $\gamma$, anti-TGF- $\beta 1$, or anti-TG2 antibodies overnight at $4^{\circ} \mathrm{C}$. After washing, the membranes were incubated with horseradish peroxidase-conjugated secondary antibodies for 1 hour. The immune complexes were visualized using an enhanced chemiluminescence kit (ECL kit, Amersham Biosciences, Little Chalfont, UK). The immunoreactive bands were analyzed using the ATTO densitography software library CS analyzer (ATTO, Tokyo, Japan). The band intensities were normalized to those of $\beta$-actin in the same samples.

\section{Statistical analyses}

All statistical analyses were performed using SPSS (version 18.0; SPSS Inc., Chicago, IL, USA). GraphPad Prism 5 (GraphPad Inc., La Jolla, CA, USA) was used to present the data. Results in various groups, reported as the median \pm standard error, were compared using Mann-Whitney U-tests. A $P$ value $<0.05$ was considered statistically significant. 


\section{ACKNOWLEDGEMENTS}

Funding: This research was supported by grants from the Ministry of Education (2014R1A1A2A16055405) and Ministry of Science \& ICT (2016M3A9E8941330) of the Republic of Korea.

\section{CONFLICTS OF INTEREST}

The authors have no conflicting interests.

\section{REFERENCES}

1. Meguid El Nahas A and Bello AK (2005) Chronic kidney disease: the global challenge. Lancet 365, 331-340

2. Huang L, Scarpellini A, Funck $M$, Verderio EA and Johnson TS (2013) Development of a chronic kidney disease model in C57BL/6 mice with relevance to human pathology. Nephron Extra 3, 12-29

3. Lorand L and Graham RM (2003) Transglutaminases: crosslinking enzymes with pleiotropic functions. Nat Rev Mol Cell Biol 4, 140-156

4. Oh K, Park HB, Byoun OJ et al (2011) Epithelial transglutaminase 2 is needed for $\mathrm{T}$ cell interleukin-17 production and subsequent pulmonary inflammation and fibrosis in bleomycin-treated mice. J Exp Med 208, 1707-1719

5. Scarpellini A, Germack R, Lortat-Jacob H et al (2009) Heparan sulfate proteoglycans are receptors for the cell-surface trafficking and biological activity of transglutaminase-2. J Biol Chem 284, 18411-18423

6. Scarpellini A, Huang L, Burhan I et al (2014) Syndecan-4 knockout leads to reduced extracellular transglutaminase-2 and protects against tubulointerstitial fibrosis. J Am Soc Nephrol 25, 1013-1027

7. Hasmim M, Messai Y, Ziani L et al (2015) Critical Role of Tumor Microenvironment in Shaping NK Cell Functions: Implication of Hypoxic Stress. Front Immunol 6, 482

8. Peng $H$, Jiang $X$, Chen $Y$ et al (2013) Liver-resident NK cells confer adaptive immunity in skin-contact inflammation. J Clin Invest 123, 1444-1456

9. Sojka DK, Plougastel-Douglas B, Yang L et al (2014) Tissue-resident natural killer (NK) cells are cell lineages distinct from thymic and conventional splenic NK cells. Elife 3, e01659
10. Victorino F, Sojka DK, Brodsky KS et al (2015) Tissue-Resident NK Cells Mediate Ischemic Kidney Injury and Are Not Depleted by Anti-Asialo-GM1 Antibody. J Immunol 195, 4973-4985

11. Law BMP, Wilkinson R, Wang $X$ et al (2017) Interferon-gamma production by tubulointerstitial human CD56(bright) natural killer cells contributes to renal fibrosis and chronic kidney disease progression. Kidney Int 92, 79-88

12. Kim SY, Jeong EJ and Steinert PM (2002) IFN-gamma induces transglutaminase 2 expression in rat small intestinal cells. J Interferon Cytokine Res 22, 677-682

13. Shi FD, Ljunggren HG, La Cava A and Van Kaer L (2011) Organ-specific features of natural killer cells. Nat Rev Immunol 11, 658-671

14. Shi FD and Van Kaer L (2006) Reciprocal regulation between natural killer cells and autoreactive $T$ cells. Nat Rev Immunol 6, 751-760

15. French AR and Yokoyama WM (2004) Natural killer cells and autoimmunity. Arthritis Res Ther 6, 8-14

16. Flodstrom-Tullberg $M$, Bryceson $Y T$, Shi FD, Hoglund $P$ and Ljunggren HG (2009) Natural killer cells in human autoimmunity. Curr Opin Immunol 21, 634-640

17. Ehl S, Nuesch $R$, Tanaka $T$, Myasaka $M$, Hengartner $H$ and Zinkernagel R (1996) A comparison of efficacy and specificity of three NK depleting antibodies. J Immunol Methods 199, 149-153

18. Jiang D, Liang J, Hodge J et al (2004) Regulation of pulmonary fibrosis by chemokine receptor CXCR3. J Clin Invest 114, 291-299

19. Vosshenrich CA, Samson-Villeger SI and Di Santo JP (2005) Distinguishing features of developing natural killer cells. Curr Opin Immunol 17, 151-158

20. Shin S, Kim YH, Cho YM et al (2015) Interpreting CD56+ and $\mathrm{CD} 163+$ infiltrates in early versus late renal transplant biopsies. Am J Nephrol 41, 362-369

21. Bloushtain N, Qimron U, Bar-llan A et al (2004) Membrane-associated heparan sulfate proteoglycans are involved in the recognition of cellular targets by NKp30 and NKp46. J Immunol 173, 2392-2401

22. Wee YM, Lim DG, Kim YH et al (2008) Cell surface modification by activated polyethylene glycol prevents allosensitization after islet transplantation. Cell Transplant 17, 1257-1269 